\title{
Transcriptome analysis of paired primary colorectal carcinoma and liver metastases reveals fusion transcripts and similar gene expression profiles in primary carcinoma and liver metastases
}

Ja-Rang Lee ${ }^{1,2+}$, Chae Hwa Kwon ${ }^{1,2+}$, Yuri Choi ${ }^{1,2+}$, Hye Ji Park ${ }^{1,2}$, Hyun Sung Kim ${ }^{2,3}$, Hong-Jae Jo ${ }^{2,3}$, Nahmgun $\mathrm{Oh}^{2,3}$ and Do Youn Park ${ }^{1,2^{*}}$

\begin{abstract}
Background: Despite the clinical significance of liver metastases, the difference between molecular and cellular changes in primary colorectal cancers (CRC) and matched liver metastases is poorly understood.

Methods: In order to compare gene expression patterns and identify fusion genes in these two types of tumors, we performed high-throughput transcriptome sequencing of five sets of quadruple-matched tissues (primary CRC, liver metastases, normal colon, and liver).

Results: The gene expression patterns in normal colon and liver were successfully distinguished from those in CRCs; however, RNA sequencing revealed that the gene expression between primary CRCs and their matched liver metastases is highly similar. We identified 1895 genes that were differentially expressed in the primary carcinoma and liver metastases, than that in the normal colon tissues. A major proportion of the transcripts, identified by gene expression profiling as significantly enriched in the primary carcinoma and metastases, belonged to gene ontology categories involved in the cell cycle, mitosis, and cell division. Furthermore, we identified gene fusion events in primary carcinoma and metastases, and the fusion transcripts were experimentally confirmed. Among these, a chimeric transcript resulting from the fusion of RNF43 and SUPT4H1 was found to occur frequently in primary colorectal carcinoma. In addition, knockdown of the expression of this RNF43-SUPT4H1 chimeric transcript was found to have a growth-inhibitory effect in colorectal cancer cells.
\end{abstract}

Conclusions: The present study reports a high concordance of gene expression in the primary carcinoma and liver metastases, and reveals potential new targets, such as fusion genes, against primary and metastatic colorectal carcinoma.

Keywords: Colorectal cancer, RNA-seq, Expression profiling, Gene fusion

\footnotetext{
* Correspondence: pdy220@pusan.ac.kr

${ }^{\dagger}$ Equal contributors

'Department of Pathology Pusan National University Hospital, Pusan National

University School of Medicine, Seo-Gu, Busan 602-739, Korea

${ }^{2}$ BioMedical Research Institute Pusan National University Hospital, Seo-Gu,

Busan, Korea

Full list of author information is available at the end of the article
} 


\section{Background}

Colorectal cancer is a commonly occurring cancer worldwide [1]. Metastatic colorectal cancer is clinically significant, as colorectal cancer is one of the major causes of cancer-related deaths [2]. Metastatic progression in colorectal cancer is a multistep process, beginning with the formation of adenomatous polyps, which develop into locally invasive tumors [3]. This process involves phenotypic changes associated with the acquisition of new functions, such as cell-type transition, cell migration, and tissue invasion in the tumor cells [3]. An improved understanding of the molecular alterations associated with metastatic progression may contribute to the development of novel and effective targeted therapies for colorectal cancer [4].

Gene expression profiling provides a scalable molecular method for investigating genetic variation, associated with ectopic gene expression, in tumors. Also, the identification of differentially expressed genes offers great potential for the discovery of clinically useful biomarkers in tumor cells. The complexity of the cancer transcriptome is attributable to differential pre-mRNA processing, including alternative promoter and splicing, which is involved in the production of cancer-specific transcripts and proteins [5]. Fusion transcripts are common cancer-specific RNAs, which are obtained by genomic rearrangements or transcription-mediated mechanisms, such as novel cis or trans splicing [6]. The formation of gene fusions may lead to the disruption of tumor suppressor genes or the activation of oncogenes, thereby triggering tumorigenesis [7]. Furthermore, fusion transcripts and proteins have been useful in cancer diagnosis, prognosis, and direct target therapy.

Massively parallel RNA sequencing (RNA-seq) is a useful method for annotation of the cancer transcriptome with great efficiency and high resolution [8] RNA-seq has enabled a comprehensive understanding of the complexity of the cancer transcriptome, via genome-wide expression profiling and identification of novel and fusion transcripts [9]. Recently, RNA-seq has been used to annotate the cancer transcriptome in breast [10], lung [11], gastric [12], and colorectal cancers $[13,14]$. However, despite the availability of high-throughput sequencing technology, the transcriptional differences including fusion genes between primary colorectal carcinomas and liver metastases not fully understood.

In this study, we compared the transcriptomes of five sets of quadruple-matched tissues (primary carcinomas, liver metastases, normal colon, and liver). First, we found a similar gene expression pattern between primary and metastatic colorectal carcinoma. Second, we identified a novel gene fusion event specifically in primary and metastatic colorectal cancer tissue, and experimentally confirmed the fusion product. In addition, we demonstrated the cell growthpromoting effect of this fusion transcript.

\section{Methods \\ Collection of specimens}

Matched fresh-frozen samples, including 5 paired primary, metastatic colorectal carcinoma, normal colon and liver, who received resection of the primary tumor at the Korean National Biobank of Pusan National University Hospital (PNUH) were obtained from the Korean National Biobank of PNUH. This series of studies was reviewed and approved by Institutional Ethics Committees of Pusan National University Hospital. All of the patients that were used in this study and their characteristics were summarized in Additional file 1: Table S1.

\section{cDNA library preparation and high-throughput paired-} end RNA sequencing

Total RNA was isolated from fresh-frozen tissues of the conditioned volunteers and patients (NC, normal colon; PC, primary colon carcinoma; LM, colon-liver metastases; NL, normal liver) using TRIzol reagents (Invitrogen, USA), and subsequently treated with RNase-free DNaseI for $30 \mathrm{~min}$ at $37^{\circ} \mathrm{C}$, to remove residual DNA. Libraries were prepared according to the standard Illumina mRNA library preparation (Illumina Inc, USA). Briefly, Purified mRNA was fragmented in fragmentation buffer and we obtained short fragments of mRNA. These short fragments served as templates to synthesize the firststrand cDNA, using random hexamer primers. The second-strand cDNA was synthesized using buffer, dNTPs, RNase $\mathrm{H}$, and DNA polymerase I, respectively. Double-stranded cDNAs were purified with QiaQuick PCR extraction kit (Qiagen Inc, USA) and resolved with EB buffer. Following the synthesis of 2nd strand, end repair, and addition of a single A base, Illumina sequencing adaptors were ligated onto the short fragments.

The concentration of each library was measured by real-time PCR. Agilent 2100 Bioanalyzer was used to estimate insert size distribution. Constructed libraries were sequenced (90 cycles) using Illumina $\mathrm{HiSeq}^{\mathrm{TM}}$ 2000 (Illumina Inc), according to the manufacturer's instructions. HiSeq Control Software (HCS v1.1.37) with RTA (v1.7.45) was used for management and execution of the HiSeq ${ }^{\mathrm{TM}} 2000$ runs.

\section{RNA-seq data processing}

Images generated by HiSeqTM2000 were converted into nucleotide sequences by a base calling pipeline and stored in FASTQ format, and the dirty raw reads were removed prior to analyzing the data. Three criteria were used to filter out dirty raw reads: Remove reads with 
sequence adaptors; Remove reads with more than $5 \%$ ' $\mathrm{N}$ ' bases; Remove low-quality reads, which have more than $50 \%$ QA $\leq 10$ bases. All subsequent analyses were based on clean reads.

Clean reads were mapped to reference Homo sapiens transcriptome sequences from the UCSC website (hg19), using Bowtie 2 and Tophat 2.0.1. Mismatches of no more than 3 bases were allowed in the alignment for each read. Reads matched with reference rRNA sequences were also mapped and removed. To annotate gene expression, fragments per $\mathrm{kb}$ per million fragments (FPKM) values of each gene were calculated, and differentially expressed genes (DEGs) were extracted using this value. The formula for calculating FPKM value was defined as below:

$$
\mathrm{FPKM}=\frac{10^{9} \mathrm{C}}{N L / 10^{3}}
$$

In this formula, $\mathrm{C}$ represents the number of reads uniquely mapped to the given gene, $\mathrm{N}$ is the number of reads uniquely mapped to all genes, and $\mathrm{L}$ is the total length of exons from the given gene. For genes with more than one alternative transcript, the longest transcript was selected to calculate the FPKM value.

\section{Expression profiling and analysis of differential gene expression}

For clustering, genes with median of RPKM $<1.0$ and coefficient of variation $(\mathrm{CV})<0.7$ were excluded to remove genes non-informative. This resulted in a total of 7744 unique genes. $\log _{2}$ transformation and additional normalization was applied. Then, hierarchical clustering was done by Gene Cluster 3.0 with default parameters, correlation (uncentered), and complete linkage [15]. The differential expression $P$-values were adjusted using the false discovery rate (FDR) by the Benjamini and Hochberg procedure and set a cutoff of FDR $<0.05$. Analyzed genes were functionally annotated in accordance with the Gene Ontology (GO) using the DAVID bioinformatics tool (http://david.abcc.ncifcrf.gov) [16].

\section{Candidate gene fusion identification}

SOAPfuse v1.26 (http://soap.genomics.org.cn/soapfuse.html) [17] was used for scanning of fusion RNAs using transcriptome data. Briefly, GRCh37.69.gtf.gz (Homo sapiens) was downloaded from Ensembl and used as gene annotation reference information (gtf). For cytoband information, the human genome (hg19, Reference 37) from UCSC, as well as the complete HGNC gene family dataset (HGNC), was used. The pipelines were tuned using Perl.

\section{Validation of fusion genes}

Fusion genes were validated by reverse transcriptionpolymerase chain reaction (RT-PCR) amplification of fusion gene breakpoints, and Sanger sequencing. The PCR reactions were carried out for $4 \mathrm{~min}$ at $94{ }^{\circ} \mathrm{C}$; $35 \mathrm{cy}$ cles of $40 \mathrm{~s}$ at $94{ }^{\circ} \mathrm{C}, 40 \mathrm{~s}$ at $55-58{ }^{\circ} \mathrm{C}$ and $40 \mathrm{~s}$ at $72{ }^{\circ} \mathrm{C}$, and finally $7 \mathrm{~min}$ at $72{ }^{\circ} \mathrm{C}$. The primer sequences are listed in Additional file 2: Table S4. PCR products were confirmed on a $2 \%$ agarose gel, purified, and cloned into the pGEM-T easy vector (Promega, USA). The positive clones were selected for Sanger sequencing. GAPDH was used as a standard control.

\section{siRNA transfection}

To suppress expression of RNF43-SUPT4H1, DLD-1 and HT29 cells were transiently transfected with siRNAs of the fusion transcript, and negative siRNA, in 6-well plates $\left(2 \times 10^{5}\right.$ cells/well). The siRNAs sequences used against the RNF43-SUPT4H1 fusion transcript variant 1 were candidate 1 in position $90 \mathrm{bp}$ : 5' -CGA CAG CGC AAC AGA CUA U-3' (sense) and 5'-AUA GUC UGU UGC GCU GUC G-3' (antisense), and candidate 2 in position 97 bp: 5'-GCA ACA GAC UAU AGA CCA G3' (sense) and 5' -CUG GUC UAU AGU CUG UUG C$3^{\prime}$ (antisense) and negative siRNA were purchased from RNAi Co. (Bioneer, Korea). These siRNA candidates targeted fusion junction (Additional file 3: Figure S5). In each colorectal cancer cell line, $100 \mathrm{nM}$ siRNA was treated using the RNAi MAX transfection reagent (Invitrogen), following the manufacturer's instructions. The cells were harvested at 24, 48 and $72 \mathrm{~h}$ after transfection, and RNF43-SUPT4H1 fusion transcript expression was analyzed by RT-PCR.

\section{MTT assay}

Cell viability was assessed by tetrazolium salt reduction using the MTT [3-(4, 5-dimethylthiazol-2-yl)-2, 5diphenyl tetrazolium bromide] assay (Sigma-Aldrich, USA). After siRNA transfection, the cells were incubated for $0,24,48$, and $72 \mathrm{~h}$ before the addition of MTT substrate. MTT stock solution was added at a final concentration of $0.5 \mathrm{mg} / \mathrm{ml}$, and cells were incubated at $37{ }^{\circ} \mathrm{C}$ for $1.25 \mathrm{~h}$. MTT crystal was collected and dissolved by incubation with DMSO. Absorbance was measured by spectrophotometry at $540 \mathrm{~nm}$ wavelength.

\section{Access to data from this study}

All RNA-seq data from this study are available for download through the NCBI Sequence Read Archive (SRA) (http://www.ncbi.nlm.nih.gov/sra), under accession number SRR2089755. 

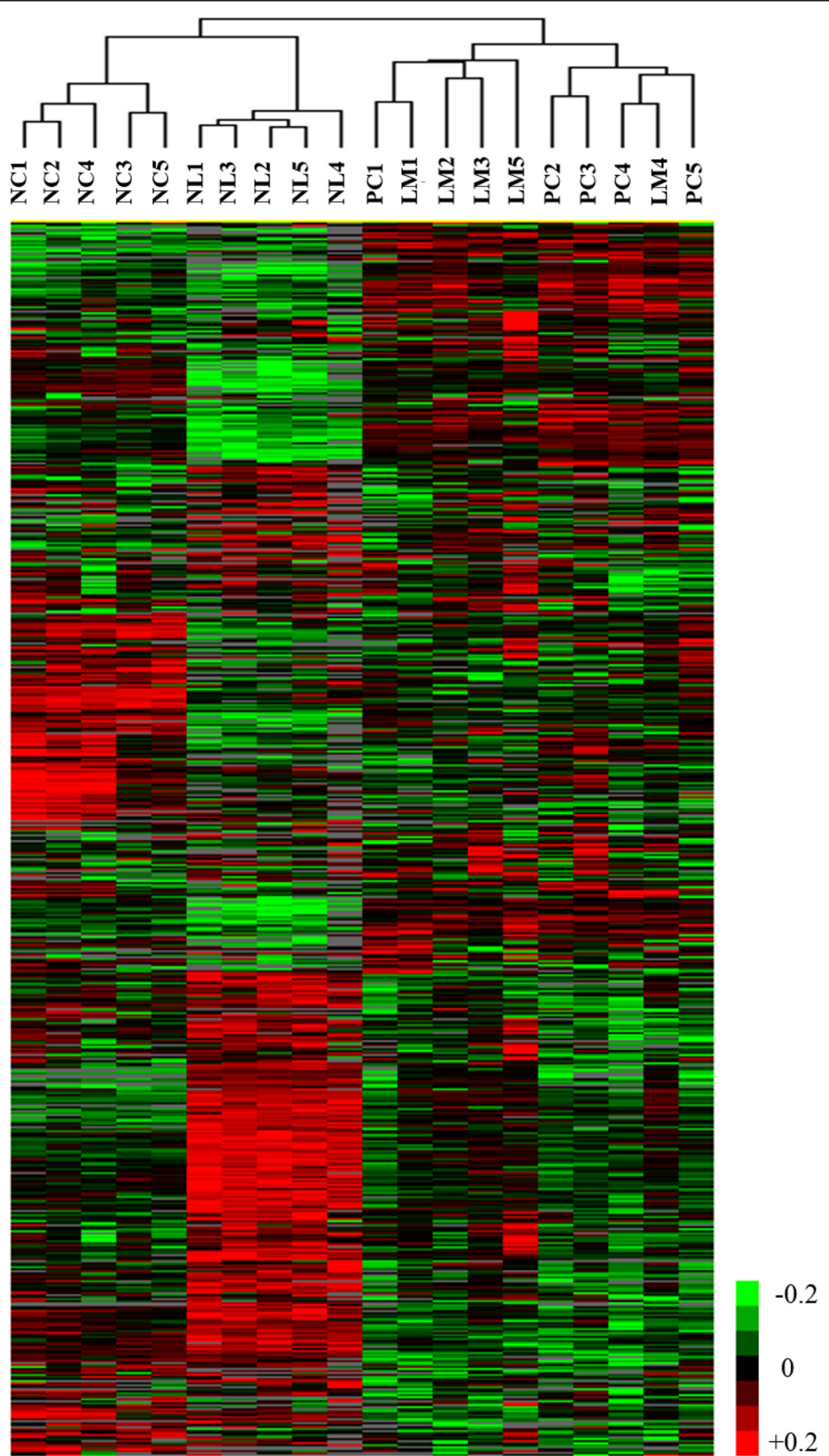

Fig. 1 Hierarchical clustering of expression profiles. Data are presented in a matrix format, in which each row represents an individual gene and each column represents a different tissue sample. Red, high expression; green, low expression. NC, normal colon; PC, primary carcinoma; LM, liver metastases; NL, normal liver 


\section{Results}

\section{Transcriptome sequencing and mapping}

Five sets of quadruple-matched tissues (primary carcinomas, liver metastases, normal colon, and liver) were collected from Pusan National University Hospital. The clinical information for patients, whose samples were used in this study, is shown in Additional file 1: Table S1. All samples were subjected to high-throughput transcriptome sequencing. About 67.3-87.1 million raw reads from each samples were sequenced (Additional file 4: Table S2). After low-quality reads were filtered out, about 88.15-92.03 \% reads were analyzed and mapped to the reference human genome $\mathrm{Hg} 19$. The average depth of coverage was $>89$ fold of that of the human transcriptome.

\section{Genes expression profiling}

The normalized expression level of each gene was expressed as Fragments Per Kilobase of Exon Per Million Fragments Mapped (FPKM). By setting a FPKM >1 threshold, we detected 56,268 reliable transcripts, which included the majority of the annotated human reference genes. We calculated the Pearson correlation coefficient to compare global gene expression between the samples. The correlation coefficients of primary carcinoma and liver metastases were higher compared to those of normal tissues (Additional file 5: Figure S2). In addition, unsupervised clustering analysis was performed. Genes with median of FPKM $<1.0$ and coefficient of variation $(\mathrm{CV})<$ 0.7 were excluded to remove genes noninformative for clustering. This resulted in a total of 7744 unique genes. The hierarchical clustering results showed that normal colon and liver were successfully distinguished from colorectal carcinoma, but primary carcinoma preferentially clustered with their matched liver metastases (Fig. 1). These results suggest a high concordance of gene expression in the primary carcinoma and liver metastases.

\section{Functional enrichment analysis of differentially expressed genes}

The common 1895 DEGs in primary carcinoma and liver metastases, compared with normal colon, were detected. In order to investigate their roles in tumor development, we performed functional enrichment analysis of DEGs using the web-based tool DAVID [16]. The common 1895 DEGs were annotated in GO component, GO function, and GO process. Among the three GO categories, "cell cycle", "cell division", and "cellular process" were dominant (Table 1). These results suggested that common DEGs are related to tumor phenotype-associated processes, such as cell cycle regulation.

We have also analyzed to select genes related to liver metastasis. We detected 694 genes differentially expressed between colorectal primary tumors and liver metastasis
Table 1 Functional annotation of differentially expressed genes (1895 gene)

\begin{tabular}{llclc}
\hline Term & Count & Percent & P-value & Benjamini \\
\hline cell cycle & 97 & 1.1 & $1.5 \mathrm{E}-36$ & $7.8 \mathrm{E}-34$ \\
mitosis & 55 & 0.6 & $7.7 \mathrm{E}-29$ & $2.0 \mathrm{E}-26$ \\
cell division & 61 & 0.7 & $3.0 \mathrm{E}-25$ & $5.2 \mathrm{E}-23$ \\
kinetochore & 25 & 0.3 & $2.0 \mathrm{E}-16$ & $2.9 \mathrm{E}-14$ \\
centromere & 17 & 0.2 & $3.6 \mathrm{E}-12$ & $3.7 \mathrm{E}-10$ \\
nucleus & 287 & 3.1 & $4.7 \mathrm{E}-12$ & $4.1 \mathrm{E}-10$ \\
phosphoprotein & 432 & 4.7 & $5.5 \mathrm{E}-11$ & $4.1 \mathrm{E}-9$ \\
DNA replication & 22 & 0.2 & $4.3 \mathrm{E}-10$ & $2.8 \mathrm{E}-8$ \\
polymorphism & 620 & 6.8 & $4.4 \mathrm{E}-9$ & $2.6 \mathrm{E}-7$ \\
microtubule & 30 & 0.3 & $1.2 \mathrm{E}-6$ & $6.1 \mathrm{E}-5$ \\
cytoskeleton & 58 & 0.6 & $1.7 \mathrm{E}-6$ & $8.0 \mathrm{E}-5$ \\
acetylation & 170 & 1.9 & $5.9 \mathrm{E}-6$ & $2.6 \mathrm{E}-4$ \\
DNA damage & 26 & 0.3 & $1.1 \mathrm{E}-5$ & $4.2 \mathrm{E}-4$ \\
cadmium & 6 & 0.1 & $1.1 \mathrm{E}-5$ & $4.0 \mathrm{E}-4$ \\
ATP-binding & 96 & 1.0 & $1.5 \mathrm{E}-5$ & $5.3 \mathrm{E}-4$ \\
DNA repair & 24 & 0.3 & $2.9 \mathrm{E}-5$ & $9.4 \mathrm{E}-4$ \\
chromosomal protein & 20 & 0.2 & $4.4 \mathrm{E}-5$ & $1.4 \mathrm{E}-3$ \\
cell cycle control & 9 & 0.1 & $6.5 \mathrm{E}-5$ & $1.9 \mathrm{E}-3$ \\
ubl conjugation & 49 & 0.5 & $1.2 \mathrm{E}-4$ & $3.2 \mathrm{E}-3$ \\
metal-thiolate cluster & 6 & 0.1 & $1.3 \mathrm{E}-4$ & $3.4 \mathrm{E}-3$ \\
Fanconi anemia & 6 & 0.1 & $2.0 \mathrm{E}-4$ & $5.0 \mathrm{E}-3$ \\
acetylated amino end & 15 & 0.2 & $2.1 \mathrm{E}-4$ & $5.1 \mathrm{E}-3$ \\
chelation & 5 & 0.1 & $2.8 \mathrm{E}-4$ & $6.3 \mathrm{E}-3$ \\
Chromosome partition & 8 & 0.1 & $4.5 \mathrm{E}-4$ & $9.8 \mathrm{E}-3$ \\
nucleotide-binding & 107 & 1.2 & $8.4 \mathrm{E}-4$ & $1.7 \mathrm{E}-2$ \\
DNA condensation & 5 & 0.1 & $1.7 \mathrm{E}-3$ & $3.4 \mathrm{E}-2$ \\
\hline & & & & \\
\hline
\end{tabular}

tumors (FDR $<0.05$, fold change $>2$ ). Of these genes, we selected 14 DEGs compared with normal colon $(\mathrm{FDR}<0.05$, fold $>2)$ and normal liver tissues $(\mathrm{FDR}<$ 0.05 , fold $>2$ ) (Additional file 6: Table S3). Most of these genes are highly expressed in normal liver and their expression in liver metastase are lower.

\section{Detection of gene fusion events}

To identify gene fusion events, SOAPfuse algorithm [17] was used. In this study, a total of 262 fusion events were found: normal colon, 74; primary carcinoma, 103; liver metastases, 67; normal liver, 71 fusion events. Gene fusion events were unique or shared among the four tissues types examined, as shown in Fig. 2. Within these gene fusion events, 73 and 36 cancer type-specific fusion events were found in the primary carcinoma and liver metastases, respectively. We focused on cancer typespecific events and gene fusions that are common in colorectal cancer, and selected fusion genes that arose 


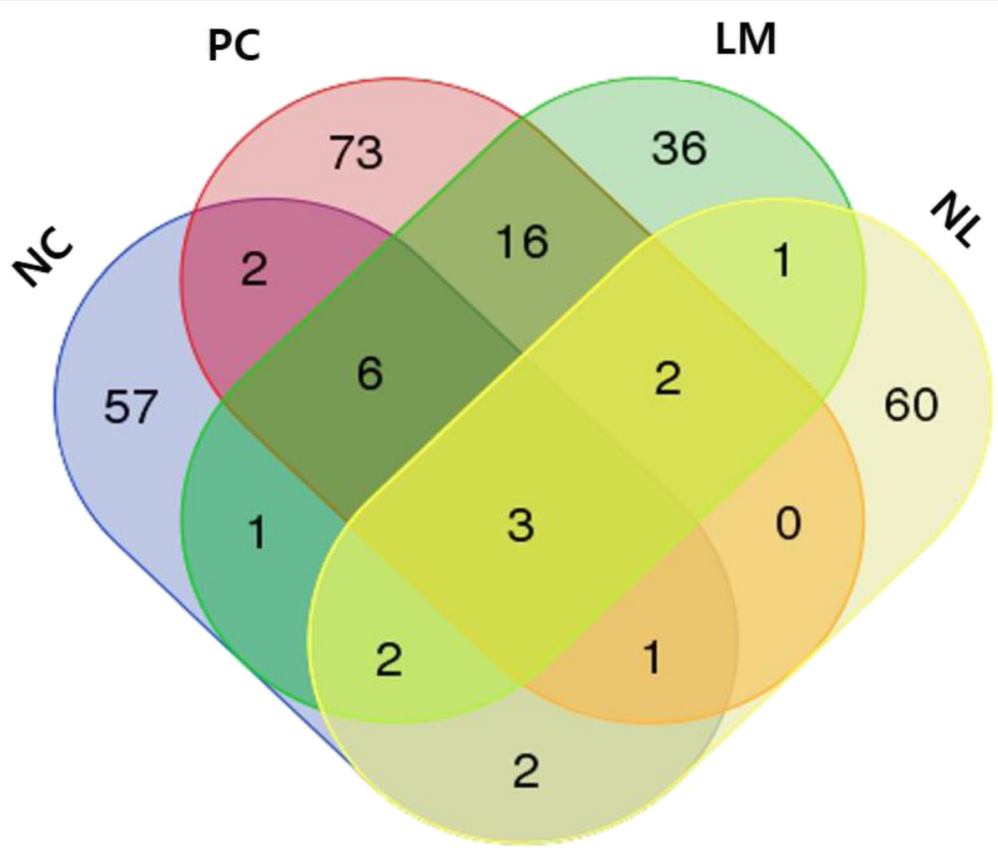

Fig. 2 The Venn diagram for comparison of gene fusion events that are unique or shared in the 4 tissue types. NC, normal colon; PC, primary carcinoma; LM, liver metastases; NL, normal liver

due to in-frame fusions (Table 2). Most fusion partner genes were located on the same chromosome, while some were formed between genes on two different chromosomes.

\section{Validation of fusion genes}

In order to experimentally confirm the gene fusions identified by RNA-Seq, three fusion transcripts were selected for validation by RT-PCR. We chose three cases of fusion events, representing inter-chromosomal and intra-chromosomal complex rearrangements, and readthrough transcription. A primer pair was designed to coordinate with the first exon of RNF43 and the exon junction, as well as the second and third exons of SUPT4H1 (Fig. 3a). The results confirmed the fusion event in the primary carcinoma, and the fusion junction was confirmed by Sanger sequencing (Fig. 3b). Also, we found an alternative fusion transcript in the primary carcinoma, which contained a part of the first exon of the SUPT4H1 gene. In addition, ZMYND8-SEPT9 and $A C E 2-P I R$ fusion transcripts were also successfully amplified by RT-PCR, and these fusion junctions were confirmed by Sanger sequencing (Additional file 7: Figure S3). These results confirmed fusion events in the sample, consistent with results of RNA-seq analysis.

To confirm the frequency of occurrence of the RNF43SUPT4H1 fusion, we screened for the expression of the fusion transcript in ten paired samples (Additional file 8: Figure S4). The RNF43-SUPT4H1 fusion transcripts were found to occur frequently, and exhibit cancer-specific expression patterns. In addition, we screened 4 colorectal cancer cell lines using fusion-specific PCR primers, for additional confirmation of frequency of the RNF43-SUPT4H1 fusion. RNF43-SUPT4H1 fusion transcripts were identified in all four colorectal cancer cell lines (Fig. 3c).

\section{Functional analysis of the RNF43-SUPT4H1 fusion gene}

In order to investigate the role of the RNF43-SUPT4H1 fusion transcript in colorectal cancer cell growth, the expression of fusion transcript variant 1 was downregulated in colorectal cancer cell lines, DLD-1 and HT29. We synthesized two candidate siRNAs against the fusion transcript. Endogenous expression of fusion transcript variant 1 was successfully inhibited in the DLD-1 and HT29 cell lines by both RNF43-SUPT4H1 candidate siRNAs (Fig. 4c, f); however, siRNA transfection had no effect on the expression of the original RNF43 and SUPT4H1 gene (Fig. 4a, b). Knockdown of fusion transcript variant 1 resulted in a significant decrease in cell proliferation at 48 and $72 \mathrm{~h}$ after transfection in the DLD-1 cell line (Fig. 4d). In the HT29 cell line, cell proliferation similarly decreased at $72 \mathrm{~h}$ after transfection (Fig. 4f). These results suggest that the RNF43-SUPT4H1 fusion transcript has a positive effect on cell growth in colorectal cancer.

\section{Discussion}

In this study, we performed transcriptome analysis using RNA-seq, to compare the gene expression profiles of primary colorectal carcinoma and liver metastases. Our 
Table 2 Summary of in-frame gene fusions

\begin{tabular}{|c|c|c|c|c|c|c|c|c|}
\hline 5'Gene & Location & Direction & 3'Gene & Location & Direction & Chimera class & Cancer type & Patients no. ${ }^{a}$ \\
\hline GTF2E2 & chr8:30510950 & - & NGR1 & chr8:32585467 & + & intrachromosomal complex & & $1(\mathrm{PC}, \mathrm{LM})$ \\
\hline TMEM66 & chr8:29940363 & - & NGR1 & chr8:32453346 & + & intrachromosomal complex & Identical patient & $4(P C, L M)$ \\
\hline TNNC2 & chr20:44452630 & - & WFDC3 & chr20:44404241 & - & intrachromosomal complex & & $3(P C, L M)$ \\
\hline HEPHL1 & chr11:93800911 & + & PANX1 & chr11:93862496 & + & intrachromosomal_NG ${ }^{\mathrm{b}}$ & & $3(P C, L M)$ \\
\hline KIAA1984 & chr9:139701518 & + & C9orf86 & chr9:139717977 & + & intrachromosomal_NG & Common fusion & $3(\mathrm{LM}) ; 4(\mathrm{PC})$ \\
\hline SLC39A1 & chr1:153933048 & - & CRTC2 & chr1:153927642 & - & intrachromosomal_NG & & $2(\mathrm{PC}) ; 4(\mathrm{LM})$ \\
\hline SCNN1A & chr12:6457893 & - & TNFRSF1A & 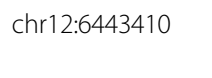 & - & intrachromosomal_NG & & $\begin{array}{l}3,5(P C) ; 1 \\
(L M)\end{array}$ \\
\hline RUVBL1 & chr3:127823674 & - & LDHA & chr11:18424445 & + & interchromosomal complex & & $2(P C)$ \\
\hline TPT1 & chr13:45914237 & - & YBX1 & chr1:43149117 & + & interchromosomal complex & & $3(P C)$ \\
\hline ZMYND8 & chr20:45976600 & - & SEPT9 & chr17:75398141 & + & interchromosomal complex & & $3(P C)$ \\
\hline $\mathrm{SOLH}$ & chr16:583998 & + & NOC4L & chr12:132635526 & + & interchromosomal complex & & $4(\mathrm{PC})$ \\
\hline CALR & chr19:13054619 & + & EEF2 & chr19:3981399 & - & intrachromosomal complex & & $5(P C)$ \\
\hline DDX27 & chr20:47855837 & + & ZNFX1 & chr20:47855592 & - & intrachromosomal complex & & $3(P C)$ \\
\hline KIF3B & chr20:30865568 & + & WFDC12 & chr20:43752906 & - & intrachromosomal complex & & $1(\mathrm{PC})$ \\
\hline GSK3B & chr3:119720893 & - & POLQ & chr3:121155122 & - & intrachromosomal complex & & $1(\mathrm{PC})$ \\
\hline CKLF & chr16:66597120 & + & CMTM1 & chr16:66611007 & + & intrachromosomal_NG & Primary specific & $2(P C)$ \\
\hline DUS4L & chr7:107217037 & + & BCAP29 & chr7:107221204 & + & intrachromosomal_NG & & $1(\mathrm{PC})$ \\
\hline HSPE1 & chr2:198367852 & + & MOBKL3 & chr2:198388348 & + & $\begin{array}{l}\text { intrachromosomal } \\
\text { complex_NG }\end{array}$ & & $2(P C)$ \\
\hline PRIM1 & chr12:57127931 & - & NACA & chr12:57118307 & - & $\begin{array}{l}\text { intrachromosomal } \\
\text { complex_NG }\end{array}$ & & $4(P C)$ \\
\hline RNF43 & chr17:56494378 & - & SUPT4H1 & chr17:56428869 & - & intrachromosomal_NG & & $4(P C)$ \\
\hline SLC10A3 & chrX:153716020 & - & UBL4A & chrX:153714672 & - & intrachromosomal_NG & & $4(P C)$ \\
\hline UBA2 & chr19:34957919 & + & WTIP & chr19:34981281 & + & intrachromosomal_NG & & $5(\mathrm{PC})$ \\
\hline ZNF606 & chr19:58499575 & - & C19orf18 & chr19:58485571 & - & intrachromosomal_NG & & $4(P C)$ \\
\hline HSP90AA1 & chr14:102551123 & - & NOP58 & chr2:203165075 & + & interchromosomal complex & & $1(\mathrm{LM})$ \\
\hline RPS15A & chr16:18794368 & - & RPLO & chr8:99057311 & - & interchromosomal complex & & $4(\mathrm{LM})$ \\
\hline FGG & chr4:155526082 & - & ALB & chr4:74285288 & + & intrachromosomal complex & & $2(\mathrm{LM})$ \\
\hline ACE2 & chrX:15582147 & - & PIR & chrX:15509432 & - & intrachromosomal complex & $\begin{array}{l}\text { Liver metastases } \\
\text { specific }\end{array}$ & $3(L M)$ \\
\hline OAF & chr11:120097705 & + & POU2F3 & chr11:120117158 & + & intrachromosomal_NG & & $2(L M)$ \\
\hline SENP3 & chr17:7474797 & + & EIF4A1 & chr17:7477578 & + & intrachromosomal_NG & & $4(\mathrm{LM})$ \\
\hline TGIF2 & chr20:35207369 & + & C20orf24 & chr20:35236118 & + & intrachromosomal_NG & & $1($ LM) \\
\hline
\end{tabular}

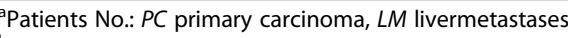

${ }^{\mathrm{b}} N G$ neighboring gene between the $5^{\prime}$ and 3 ' fusion partner

results revealed high concordance of gene expression between the primary carcinoma and liver metastases. Interestingly, we found that fusion transcripts are expressed differentially between the primary colorectal cancer and liver metastases. Our results also suggest that the fusion genes investigated may serve as potential new targets for primary colorectal carcinoma.

A recent study reported high genomic concordance between primary carcinoma and metastases in colorectal cancer $[18,19]$. In our study, the result of unsupervised clustering was in agreement with that of previous reports.
These results suggest that primary tumor and metastases may share molecular profiles at different regions. Because cancer cells that leave the primary tumor can seed metastases in distant organs $[19,20]$. However, each patient clustering showed different expression patterns between primary cancers and their metastases (Additional file 9: Figure S1). In addition, we identified 14 statistically significant genes associated with liver metastases. We will further investigate the roles of DEGs in colon cancer metastasis.

In this study, we focused on the structure of the transcriptome and analyzed cancer type-specific fusion 


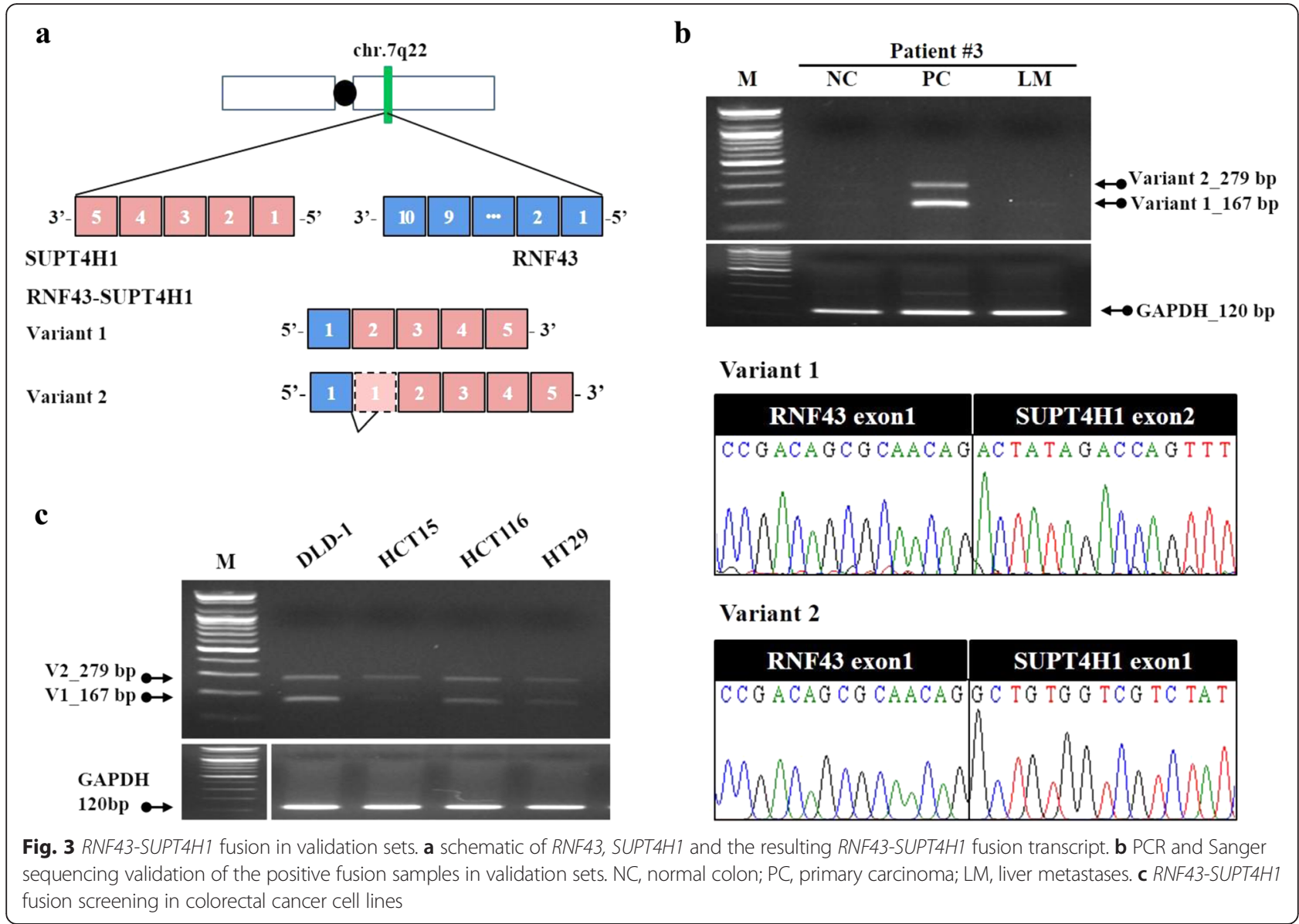

transcripts. Gene fusion events that result in genomic aberrations or transcription-mediated chimeric oncogenes are known to be involved in cancer development and progression. Fusion transcripts have been found in various cancers, including EML4-ALK in lung [21], ETV6NTRK3 in breast [22], and translocation of genes in the ETS family in prostate cancer [23]. The expression of these fusion transcripts influences cell growth, colony formation, migration, and invasion, which often results from the production of functional proteins [7]. In colorectal cancer, however, fusion transcripts are not commonly reported [24]. Investigating cancer type-specific gene fusion is useful for understanding the complexity of the cancer genome, and studying colorectal cancer development [14]. In the present study, gene fusion events in primary colorectal carcinoma and liver metastases tissues were detected using RNA-seq technique. A total of 30 inframe fusion transcripts were identified in primary carcinoma and liver metastases. Among these fusion transcripts, GTF2E2-NRG1, TMEM66-NRG1, TNNC2-WFDC, and HEPHL1-PANX1 fusion transcripts were found in both primary carcinoma and liver metastases from the same patient. It is considered that these fusion transcripts, with the exception of the HEPHL1-PANX1 gene fusion, were generated due to genomic aberrations, e.g., inversion or deletion. However, common cancer type-specific fusion transcripts are generated by transcription-mediated mechanisms, including read-through and trans-splicing, allowing for high concordance between the genomes of primary tumors and metastases. The ZMYND8-SEPT9 fusion transcript, which arises due to a fusion event involving genes on different chromosomes, is only present in primary carcinoma (Additional file 7: Figure S3A). Therefore, we suggest that the cancer type-specific fusion transcripts enable differentiation between primary carcinoma and liver metastases at the transcriptome level, regardless of genomic variation.

The Cancer Genome Atlas (TCGA) has recently reported genomic aberrations of colorectal cancer, using high-throughput sequencing [13]. The TCGA study, which focused on translocation-mediated gene fusions, reported 18 interchromosomal translocation and in-frame events. Gene fusion events may additionally occur due to genomic rearrangements. Transcription-mediated gene fusions show high frequency, and recurrent functional gene fusions are suggested as candidate biomarkers and potential therapeutic targets. We detected not only genomic rearrangement-mediated gene fusion, but also 
a

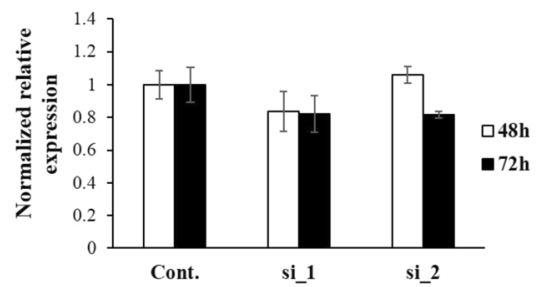

c

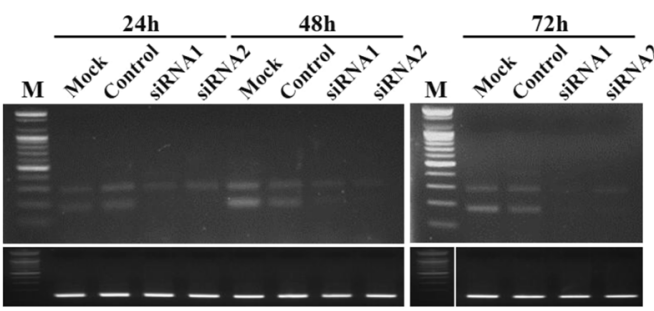

e

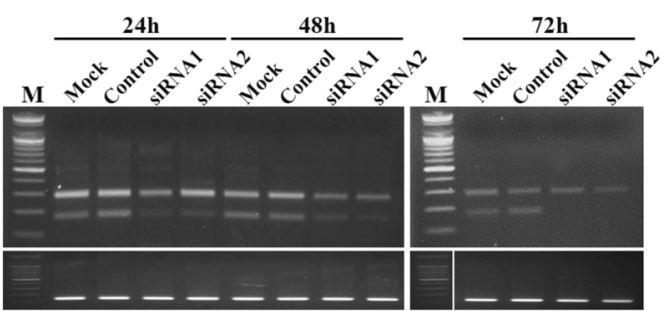

b

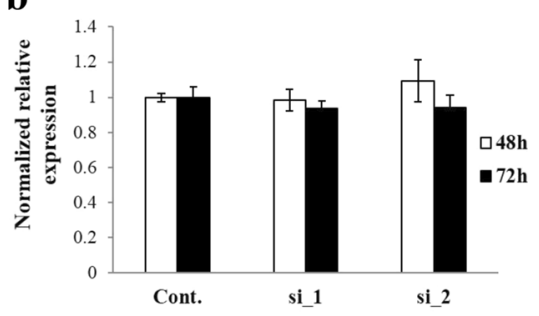

d

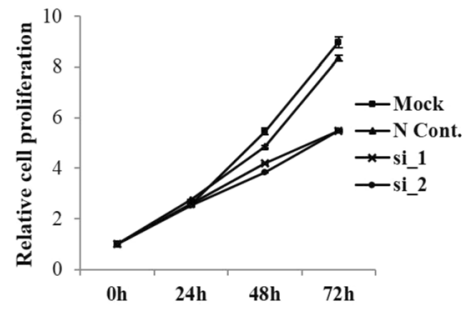

f

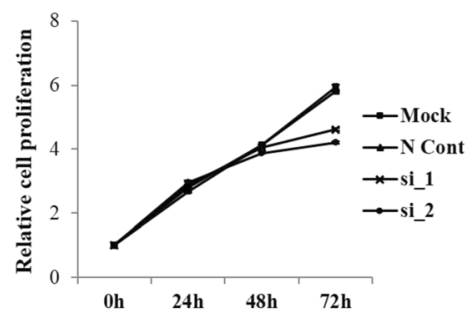

Fig. 4 Knockdown of RNF43-SUPT4H1 fusion transcript results in decreased cell proliferation. Quantitative RT-PCR of original RNF43 (a) and SUPT4H1 (b) gene in the DLD-1 cell line after transfection of siRNA targeting the RNF43-SUPT4H1 fusion transcript. c and e, RT-PCR of RNF43SUPT4H1 fusion transcript in the DLD-1 and HT29 cell line after siRNA treatment. $\mathbf{d}$ and $\mathbf{f}$, Knockdown of RNF43-SUPT4H1 fusion transcript decreased cell proliferation in the DLD-1 and HT29 cell lines

transcription-mediated gene fusion events (Table 2). Among these fusion genes, the CNN1A-TNFRSF1A fusion transcript, which is translated into fusion protein, has been reported in breast cancer [25]. Furthermore, DUS4L-BCAP29 fusion transcript has been reported in gastric cancer, which encodes a functional protein that is involved in cell proliferation [26]. We report, for the first time, that knockdown of the RNF43-SUPT4H1 fusion transcript reduces cell proliferation in live cells suggesting this fusion transcript plays a role in cancer cell growth. Therefore, we suggest that these fusion transcripts may serve as potential biomarker candidates and therapeutic targets.

The genomic loci of the RNF43 and SUPT4H1 genes are adjacent to each other, and the RNF43-SUPT4H1 fusion transcript is found to occur frequently. As a result, the RNF43-SUPT4H1 fusion transcript was categorized as a read-through chimera. This fusion transcript was detected in cancer tissues only (Fig. 3 and Additional file 8: Figure S4). We therefore hypothesized that RNF43SUPT4H1 fusion transcript acts as an oncogene, and confirmed this function (Fig. 4). RNF43 encodes the ring finger protein 43 that is involved in cell growth, and is upregulated in human colon cancer [27]. SUPT4H1 encodes the transcription elongation factor SPT4, which regulates mRNA processing and transcription elongation [28]. We speculate that the RNF43-SUPT4H1 fusion transcript is activated in colorectal cancer, affecting the expression of other genes. Future studies should focus on investigating the function of cancer type-specific fusion transcripts and developing methods for distinguishing between primary carcinoma and liver metastases.

\section{Conclusion}

This study presents the expression profiles of primary carcinoma and matched liver metastases in colorectal cancer, and reports several fusion transcripts associated with these tumor types. Although the gene expression profiles of primary carcinoma and matched liver metastases were similar, we identified cancer type-specific fusion transcripts that may be useful for distinguishing between primary carcinoma and liver metastases. These 
findings may be valuable for further studies of colorectal cancer metastasis, biomarker discovery and target identification in therapeutic drug discovery.

\section{Additional file}

Additional file 1: Table S1. Clinical information of patients used in this study.

Additional file 2: Table S4. Primer information for gene fusion validation. Additional file 3: Figure S5. RNF43-SUPT4H1 fusion transcript variant 1 targeted siRNA candidates. siRNA candidates were designed to including fusion junction. Red arrow was fusion junction, and each under bars were siRNA candidates.

Additional file 4: Table S2. Summary of statistical data for wholetranscriptome sequencing data used in this study.

Additional file 5: Figure S2. The scatter plot for global expression between samples; Pearson correlation coefficient is shown.

Additional file 6: Table S3. Genes $(n=14)$ associated with liver metastases as compared to primary tumors.

Additional file 7: Figure S3. Fusion transcripts in validation sets. (A) Gene fusion between ZMYND8 and SEPT9 gene by interchromosomal complex. (B) Gene fusion between ACE2 and PIR gene by intrachromosomal complex. Fusion junction was red arrow, and validation of fusion transcript by RT-PCR and Sanger sequencing in patient \#3. Prediction of fusion protein was analyzed by conserved domain database.

Additional file 8: Figure S4. RNF43-SUPT4H1 fusion transcript frequency in 10 paired colorectal cancer and normal tissues. M, size marker; N, normal; T, tumor.

Additional file 9: Figure S1. Hierarchical clustering of expression profiles. Data are pre-sented in a matrix format, in which each row represents an individual gene and each column represents a different tissue sam-ple. Each cell in the matrix represents the expression level of a gene feature in an individua tissue sample. Red, high expres-sion; green, low expression. N, normal colon; C, primary carci-noma; LM, liver metastases; NL, normal liver. (PPTX 1128 kb)

\section{Abbreviations}

CRC, colorectal cancers; $C V$, coefficient of variation; DEG, differentially expressed gene; FDR, false discovery rate; FPKM, fragments per kb per million fragments; GO, gene ontology; LM, colon-liver metastases; MTT, 3-(4, 5-20 dimethylthiazol-2-yl)-2, 5-diphenyl tetrazolium bromide; NC, normal colon; NL, normal liver; PC, primary colon carcinoma; RNA-seq, RNA sequencing; RT-PCR, reverse transcriptionpolymerase chain reaction; siRNA, small interfering RNA; SRA, sequence read archive; TCGA, the cancer genome atlas

\section{Acknowledgements}

The biospecimens for this study were provided by the Pusan National University Hospital, a member of the National Biobank of Korea, which is supported by the Ministry of Health, Welfare and Family Affairs. All samples derived from the National Biobank of Korea were obtained with informed consent under institutional review board-approved protocols.

\section{Funding}

This work was supported by the National Research Foundation of Korea (NRF) grant, funded by the Korean government (MSIP) (No. 2014R1A2A1A11052217).

\section{Availability of data and materials}

All RNA-seq data from this study are available for download through the NCBI Sequence Read Archive (SRA) (http://www.ncbi.nlm.nih.gov/sra), under accession number SRR2089755.

\section{Authors' contributions}

DYP designed and supervised the experiments and analyses. CHK generated sequences from the samples. CHK, JRL conducted the bioinformatics analyses. DYP, and JRL designed the validation experiments, and JRL, YRC and HJP conducted the experiments. JRL, CHK, YRC and DYP wrote the manuscript and DYP, HSK, HJJ and NO participated in improving the manuscript. All authors read and approved the final manuscript.

\section{Competing interests}

The authors declare that they have no competing interests.

Consent for publication

Not applicable.

Ethics approval and consent to participate

All patients gave written informed consent in accordance with the Declaration of Helsinki. The study was approved by the Institutional Ethics Committees of Pusan National University Hospital.

\section{Author details}

${ }^{1}$ Department of Pathology Pusan National University Hospital, Pusan National University School of Medicine, Seo-Gu, Busan 602-739, Korea. ${ }^{2}$ BioMedical Research Institute Pusan National University Hospital, Seo-Gu, Busan, Korea. ${ }^{3}$ Department of Surgery Pusan National University Hospital, Pusan National University School of Medicine, Seo-Gu, Busan, Korea.

Received: 9 September 2015 Accepted: 21 July 2016

Published online: 26 July 2016

\section{References}

1. Jemal A, Siegel R, Ward E, Hao Y, Xu J, Murray T, et al. Cancer statistics, 2008. CA Cancer J Clin. 2008;58(2):71-96.

2. Wolpin BM, Mayer RJ. Systemic treatment of colorectal cancer. Gastroenterology. 2008;134(5):1296-310

3. Nguyen DX1, Bos PD, Massagué J. Metastasis: from dissemination to organspecific colonization. Nat Rev Cancer. 2009;9(4):274-84.

4. Markowitz SD, Bertagnolli MM. Molecular origins of cancer: Molecular basis of colorectal cancer. N Engl J Med. 2009;361(25):2449-60.

5. Skotheim RI, Nees M. Alternative splicing in cancer: noise, functional, or systematic? Int J Biochem Cell Biol. 2007;39(7-8):1432-49.

6. Jividen $\mathrm{K}, \mathrm{Li} \mathrm{H}$. Chimeric RNAs generated by intergenic splicing in normal and cancer cells. Genes Chromosomes Cancer. 2014;53(12):963-71.

7. Mitelman F, Johansson B, Mertens F. The impact of translocations and gene fusions on cancer causation. Nat Rev Cancer. 2007:7(4):233-45.

8. Metzker ML. Sequencing technologies - the next generation. Nat Rev Genet. 2010;11(1):31-46.

9. Ozsolak F, Milos PM. RNA sequencing: advances, challenges and opportunities. Nat Rev Genet. 2011;12(2):87-98.

10. Eswaran J, Cyanam D, Mudvari P, Reddy SD, Pakala SB, Nair SS, et al. Transcriptomic landscape of breast cancers through mRNA sequencing. Sci Rep. 2012;2:264.

11. Cancer Genome Atlas Research Network. Comprehensive genomic characterization of squamous cell lung cancers. Nature. 2012;489(7417):519-25.

12. Cancer Genome Atlas Research Network. Comprehensive molecular characterization of gastric adenocarcinoma. Nature. 2014:513(7517):202-9.

13. Cancer Genome Atlas Research Network. Comprehensive molecular characterization of human colon and rectal cancer. Nature. 2012:487(7407):330-7.

14. Wu Y, Wang X, Wu F, Huang R, Xue F, Liang G, et al. Transcriptome profiling of the cancer, adjacent non-tumor and distant normal tissues from a colorectal cancer patient by deep sequencing. PLoS One. 2012;7(8):e41001.

15. Saldanha AJ. Java treeview-extensible visualization of microarray data Bioinformatics. 2004:20(17):3246-8.

16. Dennis Jr G, Sherman BT, Hosack DA, Yang J, Gao W, Lane HC, et al. DAVID: Database for Annotation, Visualization, and Integrated Discovery. Genome Biol. 2003;4(5):3

17. Jia W, Qiu K, He M, Song P, Zhou Q, Zhou F, et al. SOAPfuse: an algorithm for identifying fusion transcripts from paired-end RNA-Seq data. Genome Biol. 2013;14(2):R12.

18. Brannon AR, Vakiani E, Sylvester BE, Scott SN, McDermott G, Shah RH, et al. Comparative sequencing analysis reveals high genomic concordance between matched primary and metastatic colorectal cancer lesions. Genome Biol. 2014;15(8):454

19. Vignot S, Lefebvre C, Frampton GM, Meurice G, Yelensky R, Palmer G, et al. Comparative analysis of primary tumour and matched metastases in colorectal cancer patients: evaluation of concordance between genomic and transcriptional profiles. Eur J Cancer. 2015;51(7):791-9.

20. Norton L, Massagué J. Is cancer a disease of self-seeding? Nat Med. 2006; 12(8):875-8. 
21. Soda M, Choi YL, Enomoto M, Takada S, Yamashita Y, Ishikawa S, et al. Identification of the transforming EML4-ALK fusion gene in non-small-cell lung cancer. Nature. 2007:448(7153):561-6.

22. Edgren $\mathrm{H}$, Murumagi A, Kangaspeska S, Nicorici D, Hongisto V, Kleivi $\mathrm{K}$, et al. Identification of fusion genes in breast cancer by paired-end RNAsequencing. Genome Biol. 2011;12(1):R6.

23. Tomlins SA, Rhodes DR, Perner S, Dhanasekaran SM, Mehra R, Sun XW, et al. Recurrent fusion of TMPRSS2 and ETS transcription factor genes in prostate cancer. Science. 2005;310(5748):644-8.

24. Nome T, Thomassen GO, Bruun J, Ahlquist T, Bakken AC, Hoff AM, et al. Common fusion transcripts identified in colorectal cancer cell lines by highthroughput RNA sequencing. Transl Oncol. 2013;6(5):546-53.

25. Varley KE, Gertz J, Roberts BS, Davis NS, Bowling KM, Kirby MK, et al. Recurrent read-through fusion transcripts in breast cancer. Breast Cancer Res Treat. 2014:146(2):287-97.

26. Kim HP, Cho GA, Han SW, Shin JY, Jeong EG, Song SH, et al. Novel fusion transcripts in human gastric cancer revealed by transcriptome analysis. Oncogene. 2014;33(47):5434-41.

27. Yagyu R, Furukawa Y, Lin YM, Shimokawa T, Yamamura T, Nakamura Y. A novel oncoprotein RNF43 functions in an autocrine manner in colorectal cancer. Int J Oncol. 2004;25(5):1343-8.

28. Komori T, Inukai N, Yamada T, Yamaguchi Y, Handa H. Role of human transcription elongation factor DSIF in the suppression of senescence and apoptosis. Genes Cells. 2009;14(3):343-54.

\section{Submit your next manuscript to BioMed Central} and we will help you at every step:

- We accept pre-submission inquiries

- Our selector tool helps you to find the most relevant journal

- We provide round the clock customer support

- Convenient online submission

- Thorough peer review

- Inclusion in PubMed and all major indexing services

- Maximum visibility for your research

Submit your manuscript at www.biomedcentral.com/submit 